\title{
Risk factors associated with mortality and interventions in 472 neonates with interrupted aortic arch: A Congenital Heart Surgeons Society study
}

\author{
Brian W. McCrindle, MD, MPH ${ }^{\mathrm{a}}$ \\ Christo I. Tchervenkov, MD ${ }^{\mathrm{b}}$ \\ Igor E. Konstantinov, $\mathrm{MD}^{\mathrm{c}}$ \\ William G. Williams, MD ${ }^{\mathrm{c}}$ \\ Rodolfo A. Neirotti, MD \\ Marshall L. Jacobs, MD \\ Eugene $\mathrm{H}$. Blackstone, $\mathrm{MD}^{\mathrm{f}}$ \\ For the members of the Congenital Heart Surgeons Society
}

Supplementary Appendixes A through $\mathrm{H}$ are available online. $\mathcal{Z}$
From the Division of Cardiology, The Hospital for Sick Children, University of Toronto, Toronto, Ontario, Canada ${ }^{a}$; the Division of Cardiovascular Surgery, Montreal Children's Hospital, Montreal, Quebec, Canada ${ }^{\mathrm{b}}$; the Division of Cardiovascular Surgery, The Hospital for Sick Children, University of Toronto, Toronto, Ontario, Canadac; Division of Cardiothoracic Surgery, DeVos Children's Hospital, Grand Rapids, Mich ${ }^{\mathrm{d}}$; the Division of Cardiothoracic Surgery, St Christopher's Hospital for Children, Philadelphia, $\mathrm{Pa}^{\mathrm{e}}$; and the Department of Thoracic and Cardiovascular Surgery, Cleveland Clinic Foundation, Cleveland, Ohio. ${ }^{f}$

Funding of the CHSS Data Center was supplied by member surgeons and institutions and the Hospital for Sick Children (Toronto).

Read at the Eighty-fourth Annual Meeting of The American Association for Thoracic Surgery, Toronto, Ontario, Canada, April 25-28, 2004.

Received for publication April 23, 2004; revisions received Sept 4, 2004; accepted for publication Oct 1, 2004.

Address for reprints: William G. Williams, MD, The Hospital for Sick Children, 555 University Ave, Toronto, Ontario, Canada M5G 1X8 (E-mail: bill.williams@ sickkids.ca).

J Thorac Cardiovasc Surg 2005;129:343-50 $0022-5223 / \$ 30.00$

Copyright (c) 2005 by The American Association for Thoracic Surgery

doi:10.1016/j.jtcvs.2004.10.004
Objective: We sought to determine the prevalence of outcomes and associated patient and management factors for neonates with interrupted aortic arch.

Methods: From 1987 to 1997, a total of 472 neonates were enrolled prospectively from 33 institutions. Competing risks methodology was used to determine simultaneous risk and associated incremental risk factors for death, initial and subsequent left ventricular outflow tract procedures, and arch reinterventions.

Results: Overall survival was 59\% at 16 years after study entry but improved with successive birth cohort. In general, risk factors for death in each of the competing risks analyses included lower birth weight, younger age at study entry, type B interrupted aortic arch, and major associated cardiac anomalies. Of 453 patients who had interrupted aortic arch repair, after 16 years 33\% had died and 28\% had undergone an arch reintervention. Reintervention was more likely for those who had truncus arteriosus repair, interrupted aortic arch repair by a method other than direct anastomosis with patch augmentation, and the use of polytetrafluoroethylene as either an interposition graft or a patch. From study entry, competing risks after 16 years showed that $28 \%$ had died and $34 \%$ had undergone an initial left ventricular outflow tract procedure. Initial left ventricular outflow tract procedure was more likely for those with single ventricle, type B interrupted aortic arch, bicuspid aortic valve, or anomalous right subclavian artery. Among those who had undergone an initial left ventricular outflow tract procedure, after 16 years $37 \%$ had died and $28 \%$ had undergone a second procedure.

Conclusion: Anatomic features affect mortality and initial left ventricular outflow tract procedures, whereas characteristics of the arch repair affect arch reintervention.

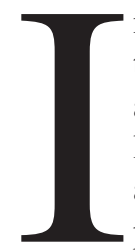

nterrupted aortic arch (IAA) occurs infrequently, and although most patients have only an isolated ventricular septal defect (VSD), more complex associated cardiovascular anomalies may occur. In addition, a spectrum of left ventricular outflow tract (LVOT) obstructive lesions, particularly subaortic stenosis, may further complicate management. Although recent reports have reflected improvements in operative survival, further interventions are often required to address residual or recurrent left heart outflow or arch repair obstructions. A previous Congenital Heart Surgeons Society (CHSS) study reported outcomes for 183 neonates entered from 1987 to $1992 .{ }^{1}$ This report builds 
on the previous report with a larger patient population, longer duration of follow-up, and inclusion of patients with major associated cardiac anomalies. It uses competing risks methodology to identify the prevalence and factors associated with death, initial and subsequent procedures directed toward actual and potential left heart outflow obstruction, and residual or recurrent obstruction of the IAA repair.

\section{Patients and Methods}

Between January 1987 and December 1997, a total of 472 neonates with IAA were admitted and enrolled by one of 33 CHSS member institutions. The number of patients enrolled per institution ranged from 1 to 57, with 15 institutions enrolling fewer than 10 patients and 8 institutions enrolling more than 20 patients. Participation by member institutions was voluntary and confidential, and ethical approval was obtained according to local requirements. Ethics approval for the CHSS Data Center is obtained annually from the Research Ethics Board of the Hospital for Sick Children, Toronto.

\section{Study Population}

All patients with IAA admitted to a CHSS institution within 30 days of birth were eligible for inclusion. IAA was defined as either a complete discontinuity or a nonpatent fibrous strand in the transverse arch or aortic isthmus.

\section{Data Collection}

Data were abstracted from submitted copies of medical records requested for initial and subsequent assessments, admissions, and procedures and entered into a database by the Data Center staff and some CHSS member surgeons. The most recent cross-sectional follow-up was performed between March and June, 2003, with 186 deaths and follow-up completed for 139 (49\%) of 286 presumed survivors. However, if cross-sectional follow-up data from the follow-up performed in 2002 are included for 28 children without 2003 follow-up, 167 (58\%) had cross-sectional follow-up and the vital status of $354(75 \%)$ patients was known within 1 year of this analysis.

\section{Data Analysis}

The goals of the analysis were to describe patient and management characteristics and to relate these and institutions as incremental risk factors in competing risks analysis of time-related mortality, LVOT procedures, and IAA repair reinterventions. Data are given as frequency, median with range, or mean $\pm \mathrm{SD}$ as appropriate, with the number of nonmissing values indicated. All data analyses were performed with SAS statistical software (version 8; SAS Institute, Inc, Cary, NC). Multiphase parametric modeling of the hazard function and competing risks methodology were used to define rates of transition to mutually exclusive time-related events and incremental risk-factors associated with each outcome, as previously described. ${ }^{2}$ Additional information regarding statistical techniques is given in Supplementary Appendix A.

\section{Results}

Patient Characteristics

Patient and morphologic characteristics are shown in Supplementary Appendix B: Table 1.

\section{Death With No Interventions}

Nineteen patients had no completed procedures, and all of these died at a median interval from admission of 11 days (range 2-45 days). Causes of death included withdrawal of support in 8 cases, cardiopulmonary arrest in 4 , complications of prematurity in 4 , and cardiac arrest during an attempted procedure in 3 . Independent factors from multiple logistic regression analysis for an increased risk of death with no completed interventions included lower and higher birth weight (polynomial function, bootstrap reliability $57 \%$ ) and need for dopamine infusion at presentation (reliability 63\%).

\section{Overall Mortality}

In total, 186 patients died. The hazard function for timerelated survival was characterized by a single early hazard phase, with survival at 16 years of 59\% (70\% confidence interval 56\%-61\%; Figure 1,A). Although a complete analysis of risk factors associated with overall mortality was not performed, improving outcomes were evident for patients born later in the study enrollment period $(P<.001$; Figure $1, B)$.

\section{Interventions Before, With, and After IAA Repair}

Only 5 patients had procedures performed before IAA repair. Repair of IAA was performed in 453 patients at a median age of 7 days (range 1-60 days). In 2 patients, IAA repair occurred at the time of primary heart transplantation. Characteristics of IAA repair are given in Supplementary Appendix C: Table 2, and these variables were used in multivariable analyses. A flow chart of the number of patients having initial and additional interventions aimed at potential or actual LVOT obstruction, reinterventions for residual or recurrent arch obstruction, and subsequent mortality is given in Supplementary Appendix D: Electronic Figure 1.

\section{Competing Risks for Intervention for Arch Repair Obstruction}

Among the 453 patients who underwent repair of IAA, this was followed by death in 143 cases and survival to a reintervention for IAA repair obstruction in 109 cases. Of the 109 arch reinterventions, 52 were by transcatheter balloon dilation and 57 were surgical. The time-related hazard function for survival to an IAA repair intervention showed two phases, an early phase accounting for 89 events and a smaller constant hazard phase accounting for 20 events. The time-related hazard function for death without an IAA repair intervention was characterized by an early phase only. The competing risks for the two events showed that 16 years after IAA repair, 33\% had died without an IAA repair intervention, 29\% were surviving to an IAA repair intervention, and 38\% remained alive without an IAA repair intervention (Figure 2). Incremental risk factors from mul- 


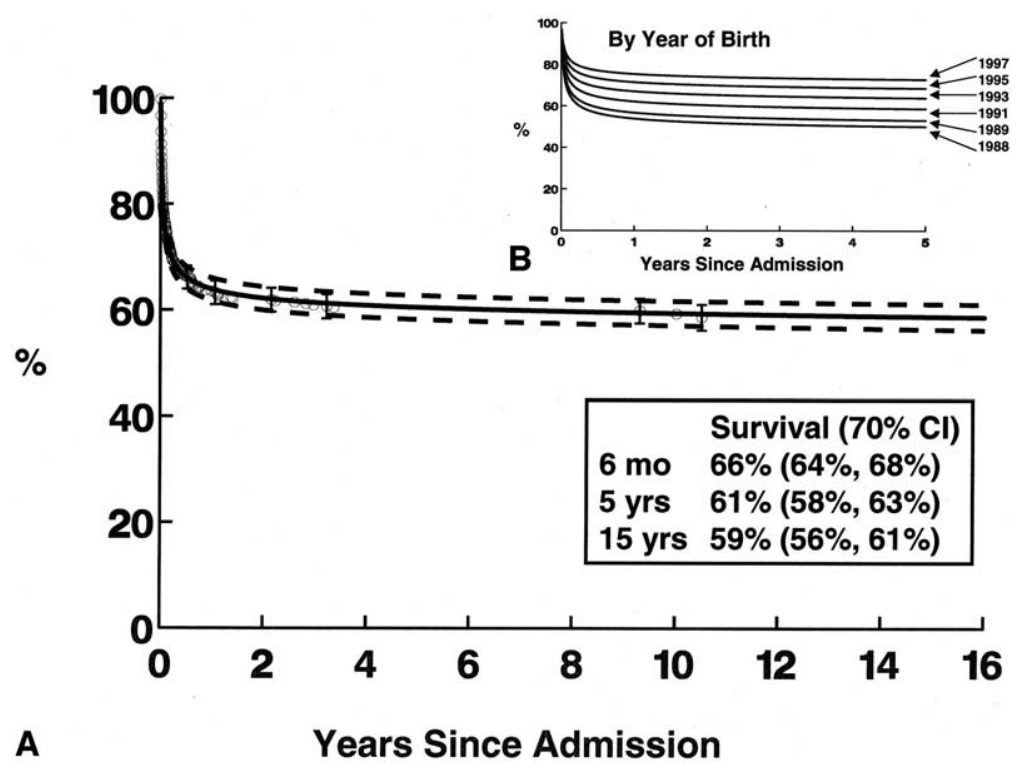

Figure 1. Overall time-related survival of 472 neonates with IAA. All patients began at time of initial admission to CHSS member institution. Solid lines represent parametric point estimates; dashed lines enclose $70 \%$ confidence intervals (CI); circles with error bars represent nonparametric estimates. A, Overall survival. B, Predicted overall survival for first 5 years after admission stratified by patient date of birth.

tivariable hazard modeling of each outcome, both inclusive and exclusive of institutions, are given in Supplementary Appendix E: Table 3.

To determine the impact of type of arch repair on subsequent outcomes, the multivariable competing risk model was then solved for various combinations of characteristics. Patient variables were set to mean values, except for type B IAA with no associated anomalies. Prediction for such a patient had repair been accomplished with a direct anastomosis with patch augmentation showed that at 16 years after repair, there was a $23 \%$ chance of survival to arch reintervention, an $18 \%$ chance of death, and a $59 \%$ chance of survival without reintervention. For repair by direct anastomosis without patch augmentation, at 16 years, there was a $24 \%$ chance of survival to arch reintervention, an increased chance of death at $30 \%$, and a chance of survival without reintervention of $47 \%$. For repair accomplished by placement of a polytetrafluoroethylene (PTFE) interposition graft with no arch augmentation, at 16 years, there was a $54 \%$ chance of survival to arch reintervention, a $30 \%$ chance of death, and a $16 \%$ chance of survival without reintervention.

\section{Competing Risks for Initial Intervention for LVOT Obstruction}

From admission to a CHSS institution, an initial intervention aimed at dealing with potential or actual LVOT obstruction was performed in 143 patients, with another 128 patients dying without such an intervention. The initial intervention was performed at the time of IAA repair for 91

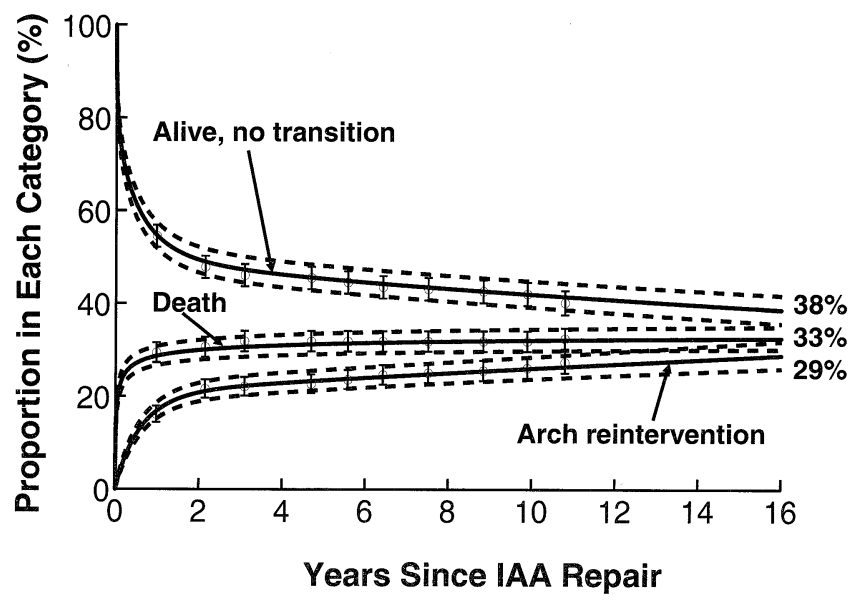

Figure 2. Competing risks analysis for subsequent intervention for residual or recurrent obstruction at IAA repair site. All patients began at time of repair of IAA $(n=453)$ and could transition to either death or subsequent intervention for residual or recurrent obstruction at arch repair site. Solid lines represent parametric point estimates; dashed lines enclose $70 \%$ confidence intervals; circles with error bars represent nonparametric estimates. Proportion of patients (expressed as percentage of total) in each of 3 categories at any given time after IAA repair.

patients. The types of interventions are given in Supplementary Appendix F: Table 4, together with the number of patients who underwent a subsequent additional LVOT intervention or who died without further LVOT interven- 


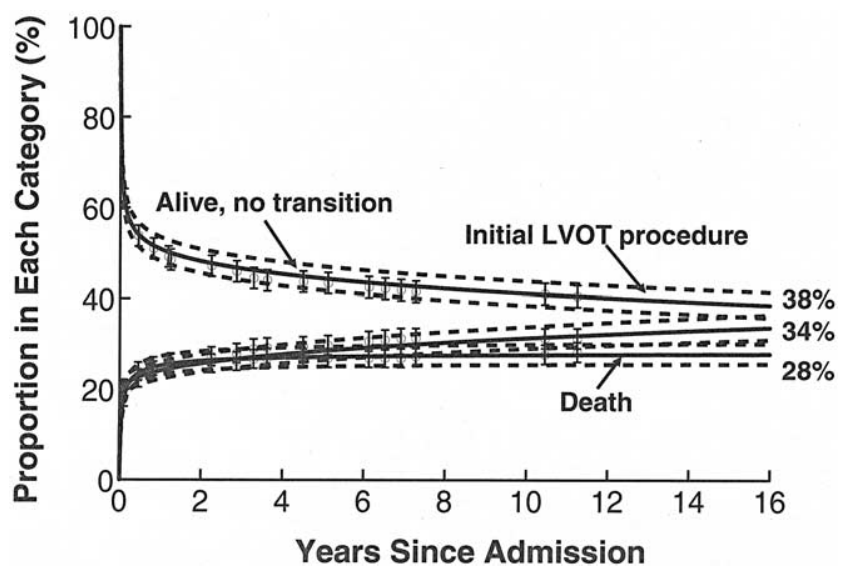

Figure 3. Competing risks analysis for initial procedure aimed at addressing potential or actual LVOT obstruction. All patients began at time of initial admission to CHSS member institution ( $n$ $=472$ ) and could transition to either death or initial procedure aimed at addressing potential or actual LVOT obstruction. Solid lines represent parametric point estimates; dashed lines enclose $70 \%$ confidence intervals; circles with error bars represent nonparametric estimates. Proportion of patients (expressed as percentage of total) in each of 3 categories at any given time after IAA repair.

tion. The hazard function for time-related transition to an initial LVOT intervention was characterized by a steep early hazard phase (90 early phase-events) and a prolonged decreasing late hazard phase (53 late-phase events). The hazard function for transition to death without an initial LVOT intervention was characterized by a steep early hazard phase only. The competing risks for the two events showed that after 16 years from admission, $28 \%$ had died and $38 \%$ were surviving without an LVOT intervention, with $34 \%$ surviving to an initial LVOT intervention (Figure 3). Incremental risk factors from multivariable hazard modeling of each outcome, both exclusive and inclusive of institutions, are given in Supplementary Appendix G: Table 5. For death without an initial LVOT intervention, the relationships with date of birth, birth, weight and age at admission were complex.

To determine the impact of major anatomic risk factors on freedom from initial LVOT procedure or death, the multivariable competing risk model was then solved for various combinations of characteristics. Patient variables, except for the anomalies of interest, were set to mean values. Prediction for a patient with isolated type B IAA with an anomalous right subclavian artery showed that at 16 years after admission, there was a $30 \%$ chance of survival to an initial LVOT procedure, a $27 \%$ chance of death, and a $43 \%$ chance of survival without an LVOT procedure. In contrast, for a patient with single ventricle, at 16 years, there was a $91 \%$ chance of survival to an initial LVOT procedure (usually creation of a main pulmonary artery-aorta connection, although 3 patients had some sort of subaortic resec-

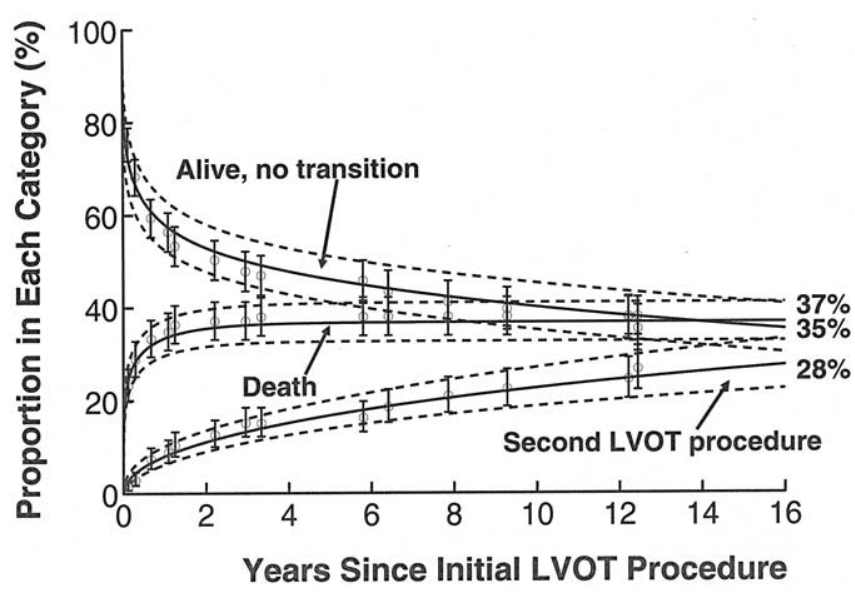

Figure 4. Competing risks analysis for subsequent procedure aimed at addressing residual or recurrent LVOT obstruction. All patients began at time of initial procedure aimed at addressing potential or actual LVOT obstruction ( $n=143$ ) and could transition to either death or similar subsequent procedure. Solid lines represent parametric point estimates; dashed lines enclose $\mathbf{7 0} \%$ confidence intervals; circles with error bars represent nonparametric estimates. Proportion of patients (expressed as percentage of total) in each of 3 categories at any given time after IAA repair.

tion), a 9\% chance of death, and almost no chance of survival without an LVOT procedure. For a patient with associated truncus arteriosus, at 16 years, there was an $18 \%$ chance of survival to an initial LVOT, a $56 \%$ chance of death, and a $26 \%$ chance of survival without an LVOT procedure.

\section{Competing Risks for Subsequent Intervention for Recurrent or Residual LVOT Obstruction After Initial Intervention}

Of the 143 patients who underwent an initial intervention, 28 survived to an additional LVOT intervention, most commonly a Konno procedure, and 53 died without subsequent intervention. The hazard function for time-related transition to a subsequent LVOT intervention was characterized by prolonged and gradually declining late hazard phase only. The hazard function for death without a subsequent LVOT intervention was characterized by a steep early phase only. The competing risks for the two events showed that after 16 years from initial LVOT intervention, 37\% had died, 35\% were surviving without a subsequent LVOT intervention, and $28 \%$ survived to a subsequent LVOT intervention (Figure 4). Incremental risk factors from multivariable hazard modeling of each outcome, both inclusive and exclusive of institutions, are given in Supplementary Appendix H: Table 6.

To determine the impact of type of initial LVOT procedure as a risk factor for freedom from a second LVOT 
procedure or death, the multivariable competing risk model was then solved for various combinations of characteristics. Patient variables, except for procedural variables, were set to mean values. Prediction for a patient whose initial LVOT procedure was balloon dilation of the LVOT showed that at 16 years after the initial procedure, there was an $84 \%$ chance of survival to a second LVOT procedure, a $16 \%$ chance of death, and almost no chance of survival without a second procedure. For a patient with an initial Norwood procedure, there was a $17 \%$ chance of survival to a second LVOT procedure (2 patients with favorable anatomy survived to undergo a subsequent conal resection and biventricular repair), a 50\% chance of death, and a 33\% chance of survival without a second procedure.

\section{Predicted Outcomes for a Realistic Patient With an Optimal Risk Factor Profile}

Given the complexity of patient characteristics and management, the multivariable competing risks analyses were solved to predict what the outcomes might be for a realistic patient with an optimal risk factor profile, to determine what might be achieved if all risk factors could be neutralized. This patient was defined as a male born in 1997 with a birth weight of $3.5 \mathrm{~kg}$ who was admitted to a non-high-risk CHSS member institution at 4 days after birth. This patient would have a single, large, malaligned VSD closed at the time of IAA repair, which was achieved with a direct anastomosis with a non-PTFE patch augmentation, and no associated anomalies. Prediction for arch reintervention would show that at 16 years after repair, he would have a $24 \%$ chance of survival to reintervention, a $7 \%$ chance of death, and a $69 \%$ chance of survival without reintervention. Prediction for an initial LVOT procedure would show that at 16 years after admission, he would have a $16 \%$ chance of surviving to an LVOT procedure, a 5\% chance of death, and a $79 \%$ chance of survival without a procedure. If he did undergo an initial LVOT procedure, he would be predicted after 16 years to have a $29 \%$ chance of survival to a second procedure, a $6 \%$ chance of death, and a $65 \%$ chance of survival without a second procedure.

\section{Discussion}

\section{Overall Mortality}

Although overall mortality was high, there was a significant and important improvement with time in our series. The previous CHSS report noted survival at 4 years after IAA repair of $63 \%$, although the analysis was limited to those patients with associated VSD. ${ }^{1}$ Risk factors for time-related death included lower birth weight, younger age at repair, type B IAA, outlet and trabecular VSD locations, smaller size of the VSD, and the presence of subaortic narrowing, together with some individual institutions. Procedural risk factors included failure to address LVOT obstruction at the time of repair, a Damus-Kaye-Stansel procedure, and use of subaortic myectomy or myotomy to address subaortic narrowing.

There have been many case reports but few case series reporting outcomes of IAA. An early report by Sell and colleagues $^{3}$ of 63 patients operated on from 1974 to 1987 showed an overall survival of $47 \%$ at 10 years. Fulton and coworkers ${ }^{4}$ reported an overall survival of $85 \%$ at 12 years after IAA repair in 72 patients operated on from 1985 to 1997, with the only significant risk factor for death being a longer duration of cardiopulmonary bypass. Serraf and colleagues ${ }^{5}$ studied 82 patients seen from 1985 to 1995 and reported an overall survival of $70 \%$ at 5 years, with similar survivals for those with simple and complex lesions but decreased survival for those with subaortic obstruction. Survival did not differ between single- and 2-stage repairs. Schreiber and associates ${ }^{6}$ reported an overall survival of $67 \%$ at 10 years after repair for 94 patients operated on from 1975 to 1999, with LVOT obstruction and a 2-stage approach significant risk factors for early mortality. Tlaskal and coworkers ${ }^{7}$ studied 40 patients who underwent procedures from 1977 to 1997, with 50\% dying within 30 days of surgery. Risk factors for early mortality included preoperative complications, acidosis, poor clinical condition, and earlier year of surgery, with better survival for those undergoing primary repair rather than palliation. Similar to our series, others have also reported a small proportion of patients who either died or had support withdrawn and did not undergo repair.

In our competing risks analysis, several variables were consistently associated with an increased mortality; these included truncus arteriosus, IAA type $\mathrm{B}$ or $\mathrm{C}$, younger age at study entry, lower birth weight, and female sex. For death after an initial LVOT procedure or after IAA repair, the procedural characteristics were also predictive. Although some early reports favored a staged approach, ${ }^{8-10}$ the subsequent trend has been toward primary repair ${ }^{11-13}$ with selective use of the staged approach. ${ }^{7}$ Death after an initial procedure for LVOT obstruction was more likely if the procedure included pulmonary artery band or shunt placement or a Norwood procedure, indicating the elements of staged repairs associated with increased mortality. Death after IAA repair was also predicted by some procedural factors, including creation of a Damus-Kaye-Stansel shunt and not augmenting the arch repair. This would suggest that more severe LVOT obstruction requiring bypass procedures and possibly failure to relieve arch obstruction contribute to mortality. Features and management of the VSD were additional significant risk factors for mortality. Absence of malalignment of the VSD, small size of the VSD, and the presence of multiple VSDs predicted death; these may be a marker for the presence of associated complex cardiac anomalies, whereas malalignment is known to be a mech- 
anism for LVOT obstruction in those with less complex lesions.

\section{Method of IAA Repair and Subsequent Arch Procedures}

Factors significantly associated with an increased risk of intervention for residual or recurrent arch obstruction included younger and older age at study entry, associated aortopulmonary window or double-outlet right ventricle, two particular institutions, and institutions contributing fewer than 10 patients, all of which were associated with early reintervention. In addition, procedural risk factors influenced late reinterventions, including repair of truncus arteriosus, use of PTFE material in the arch repair (all interposition grafts and 6 of the patch augmentations), and repair of the arch by a method other than direct anastomosis with patch augmentation. Obstruction of the arch repair has not been optimally studied, and reports are largely descriptive. Fulton and coworkers ${ }^{4}$ noted a freedom from reintervention for arch obstruction of $83 \%$ at 12 years after repair. Sell and colleagues ${ }^{3}$ reported that $47 \%$ of early survivors, more commonly those with a direct anastomosis, had arch obstruction develop. Serraf and colleagues ${ }^{5}$ noted recurrent arch obstruction in 27\%, with no relation to the type of initial arch repair. The management of arch obstruction depends on the type of initial repair. Interposition grafts and bypass conduits require surgical management, whereas anastomotic narrowings can be effectively addressed by transcatheter balloon dilatation, albeit with a risk of subsequent recurrence. ${ }^{14}$ Our data would suggest that where possible the preferred method of arch repair is by direct anastomosis augmented with either homograft or pericardial material, giving the best survival free of reintervention.

\section{LVOT Obstruction}

LVOT obstruction can occur from subaortic narrowing, aortic valve hypoplasia or stenosis, and ascending aorta hypoplasia, and it can greatly complicate patient management. We noted that patients who underwent initial LVOT procedures had lower birth weight, type B IAA, single ventricle, bicuspid aortic valve, or anomalous right subclavian artery. Similar to our series, both Fulton and coworkers $^{4}$ and Geva and associates ${ }^{15}$ noted that the presence of type B IAA and anomalous right subclavian artery were risk factors for LVOT obstruction, presumably because of alterations in blood flow across the LVOT. Of note, two institutions were associated with a greater likelihood of an initial LVOT procedure. Fulton and coworkers ${ }^{4}$ also noted that the presence of LVOT obstruction did not influence overall survival and that most did not require an intervention. Serraf and colleagues ${ }^{5}$ documented catch-up growth of the LVOT. However, we noted that among 143 patients who underwent an initial LVOT procedure, 52 procedures were as additional operations after IAA repair. Nonetheless, some pa- tients who require an initial LVOT bypass procedure can go on to have biventricular repair. ${ }^{16}$

The heterogeneity of LVOT obstructions mandates individual management. ${ }^{17,18}$ Clearly, for some patients the decision of when and how to intervene is difficult. Salem and colleagues ${ }^{19}$ performed a detailed review of echocardiographic measurements, and showed that an absolute diameter of the aortic valve annulus less than $4.5 \mathrm{~mm}$ and a $Z$ score less than -5 completely predicted the development of LVOT obstruction after arch repair, whereas size of the subaortic region did not. ${ }^{19}$ Likewise, catch-up growth of the aortic valve annulus was demonstrated. In contrast, Apfel and coworkers ${ }^{20}$ showed that echocardiographic measurements of the subaortic region predicted LVOT obstruction. Geva and associates ${ }^{15}$ found an indexed cross-sectional area of the LVOT less than $0.7 \mathrm{~mm} / \mathrm{m}^{2}$ but not aortic valve dimensions to predict LVOT obstruction. Careful quantitative echocardiographic assessment of the LVOT structures can assist decision making. Unfortunately, these data were not available in our study.

Unlike previous reports, we identified factors associated with a subsequent LVOT intervention after an initial procedure. Because of the heterogeneity of obstruction and the methods by which it can be addressed, only absence of a large VSD, initial transcatheter balloon dilation of the LVOT, and one institution (protective) were significantly associated with a second LVOT procedure. Although balloon dilatation can be an effective method of addressing isolated aortic valve stenosis, it is inadequate to address aortic valve hypoplasia and subaortic obstruction, which are more likely in the setting of IAA.

\section{Summary}

Management of IAA remains complex, although overall mortality appears to be improving. Patients with low birth weight, immediate presentation, type B IAA, and major associated cardiac anomalies remain at high risk for death and initial LVOT procedures. Although this issue was not addressed in our study, predictors of which patients will require LVOT procedures for potential or actual obstruction are difficult to identify except in specific circumstances because of the wide spectrum of mechanisms, severity, and natural history. If the initial procedure required is an LVOT bypass procedure, this marks a subgroup at high-risk for death, with more complex subsequent management. For patients who undergo balloon dilatation of the LVOT, there is a high risk of residual or recurrent obstruction requiring additional procedures. The optimal method of repair of IAA appears to be with direct anastomosis with non-PTFE patch augmentation, which reduces subsequent mortality but has a minimal impact on reducing need for reintervention. The use of an interposition graft appears to necessitate latephase reintervention in survivors. Even if the risk factors 
identified in this study can be neutralized, approximately a $7 \%$ mortality will probably remain, largely occurring early; although this was seen at the end of the era studied, which may not completely reflect contemporary results.

We thank all the members of the CHSS and their pediatric cardiology colleagues for their contributions to this study. We acknowledge the assistance of personnel from the CHSS Data Center, particularly Geraldine Cullen-Dean, Sally Cai, and Olga Levesque, for coordinating the collection and management of these data and Dr Tara Karamlou for assisting in editing the manuscript.

\section{References}

1. Jonas RA, Quaegebeur JM, Kirklin JW, Blackstone EH, Daicoff G. Outcomes in patients with interrupted aortic arch and ventricular septal defect. A multiinstitutional study. Congenital Heart Surgeons Society. J Thorac Cardiovasc Surg. 1994;107:1099-1109.

2. Blackstone EH, Naftel DC, Turner ME Jr. The decomposition of time-varying hazard into phases, each incorporating a separate stream of concomitant information. J Am Stat Assoc. 1986;81:615-24.

3. Sell JE, Jonas RA, Mayer JE, Blackstone EH, Kirklin JW, Castaneda AR. The results of a surgical program for interrupted aortic arch. J Thorac Cardiovasc Surg. 1988;96:864-77.

4. Fulton JO, Mas C, Brizard CP, Cochrane AD, Karl TR. Does left ventricular outflow tract obstruction influence outcome of interrupted aortic arch repair? Ann Thorac Surg. 1999;67:177-81.

5. Serraf A, Lacour-Gayet F, Robotin M, Bruniaux J, Sousa-Uva M, Roussin R, et al. Repair of interrupted aortic arch: a ten-year experience. J Thorac Cardiovasc Surg. 1996;112:1150-60.

6. Schreiber C, Eicken A, Vogt M, Gunther T, Wottke M, Thielmann M, et al. Repair of interrupted aortic arch: results after more than 20 years. Ann Thorac Surg. 2000;70:1896-99.

7. Tlaskal T, Hucin B, Hruda J, Marek J, Chaloupecky V, Kostelka M, et al. Results of primary and two-stage repair of interrupted aortic arch. Eur J Cardiothorac Surg. 1998;14:235-42.

8. Mainwaring RD, Lamberti JJ. Mid- to long-term results of the twostage approach for type B interrupted aortic arch and ventricular septal defect. Ann Thorac Surg. 1997;64:1782-5.

9. Irwin ED, Braunlin EA, Foker JE. Staged repair of interrupted aortic arch and ventricular septal defect in infancy. Ann Thorac Surg. 1991; 52:632-6.

10. Qureshi SA, Maruszewski B, McKay R, Arnold R, West CA, Hamilton DI. Determinants of survival following repair of interrupted aortic arch in infancy. Int $J$ Cardiol. 1990;26:303-12.

11. Luciani GB, Ackerman RJ, Chang AC, Wells WJ, Starnes VA. Onestage repair of interrupted aortic arch, ventricular septal defect, and subaortic obstruction in the neonate: a novel approach. $J$ Thorac Cardiovasc Surg. 1996;111:348-58.

12. Vouhe PR, Mace L, Vernant F, Jayais P, Pouard P, Mauriat P, et al. Primary definitive repair of interrupted aortic arch with ventricular septal defect. Eur J Cardiothorac Surg. 1990;4:365-70.

13. Tlaskal T, Chaloupecky V, Marek J, Hucin B, Kostelka M, Tax P, et al. Primary repair of interrupted aortic arch and associated heart lesions in newborns. J Cardiovasc Surg (Torino). 1997;38:113-8.

14. McCrindle BW, Jones TK, Morrow WR, Hagler DJ, Lloyd TR, Nouri $\mathrm{S}$, et al. Acute results of balloon angioplasty of native coarctation versus recurrent aortic obstruction are equivalent. J Am Coll Cardiol. 1996;28:1810-7.

15. Geva T, Hornberger LK, Sanders SP, Jonas RA, Ott DA, Colan SD. Echocardiographic predictors of left ventricular outflow tract obstruction after repair of interrupted aortic arch. J Am Coll Cardiol. 1993; 22:1953-60.

16. Erez E, Tam VK, Kanter KR, Fyfe DA. Successful biventricular repair after initial Norwood operation for interrupted aortic arch with severe left ventricular outflow tract obstruction. Ann Thorac Surg. 2001;71: 1974-7.
17. Shiokawa Y, Becker AE. The surgical anatomy of the left ventricular outflow tract in hearts with ventricular septal defect and aortic arch obstruction. Ann Thorac Surg. 1998;65:1381-7.

18. Jacobs ML, Chin AJ, Rychik J, Steven JM, Nicolson SC, Norwood WI. Interrupted aortic arch. Impact of subaortic stenosis on management and outcome. Circulation. 1995;92(9 Suppl):II128-31.

19. Salem MM, Starnes VA, Wells WJ, Acherman RJ, Chang RK, Luciani $\mathrm{GB}$, et al. Predictors of left ventricular outflow obstruction following single-stage repair of interrupted aortic arch and ventricular septal defect. Am J Cardiol. 2000;86:1044-7, A11.

20. Apfel HD, Levenbraun J, Quaegebeur JM, Allan LD. Usefulness of preoperative echocardiography in predicting left ventricular outflow obstruction after primary repair of interrupted aortic arch with ventricular septal defect. Am J Cardiol. 1998;82:470-3.

\section{Discussion}

Dr Ralph S. Mosca (New York, NY). IAA is a relatively rare, morphologically heterogeneous lesion with complexities that can be overlooked in its simplistic name. Today's excellent presentation builds on a previous study, also from the members of the CHSS. The first study confirmed the utility of a single-stage approach. The importance of multiple levels of obstruction in the left heart complex was stressed, but subaortic myomectomy and a Damus-Kaye-Stansel procedure were both risk factors for death. As shown again today, reoperative procedures were common and yielded variable results.

This morning's analysis used competing risk methodology to determine simultaneous risk and associated incremental risk for death and reintervention. McCrindle and coworkers have made it clear that anatomic features affect mortality and the need for LVOT interventions, whereas the type of arch repair determines the need for reintervention on the arch. This is an elegant analysis, but it falls short, I believe, of answering some key questions pertaining to the need for additional and subsequent reintervention. This is not a criticism of the statistical technique; rather, perhaps it reflects a lack of important preoperative data.

For example, a number of patients died from what appears to be inadequate LVOTs, both with and without intervention. Dr McCrindle, your analysis suggests that patients with more severe LVOT obstruction were at additional risk for mortality. Much of the discussion concerning the care of this lesion concerns the need for an optimal method of relieving the subaortic obstruction. Were there no data available to try to relate the size and structure of the subaortic LVOTs to initial intervention and subsequent outcomes, and can we expect that in the future updates?

Dr McCrindle. First of all, with the original article from the CHSS, there were some limited data available for some of the measurements of different LVOT structures. However, that data collection ended at a certain point, an analysis was performed, and then the study was reactivated. The subsequent data collection didn't focus on collecting these kinds of variables. The answer would likely require a detailed and quantitative analysis of initial echocardiograms. We previously published a study of patients with critical aortic stenosis in which we were able to obtain the initial echocardiogram for a lot of those patients. Logistically, however, this type of data collection, from an institutional research ethics review board standpoint, is becoming more and more problematic and more and more difficult to do. Part of the challenge relates to interpretation and compliance with the new Health Insurance Portability and Accountability Act guidelines, which restrict and regulate access to that type of data. 
However, if you examine the literature regarding studies of IAA that have incorporated some of those echocardiographic measurements, there appears to be no real consistency regarding which structures, measurements, and cut points most optimally predict whether someone has LVOT obstruction that needs to be addressed at the time of an arch repair.

Dr Mosca. I agree. That is why I was hoping we would have seen it here, but I understand your concerns.

The association of truncus arteriosus with IAA was a risk factor for both reintervention and death. Truncus obviously brings along its own intricacies but is usually free of LVOT obstruction. What is it about the association of truncus with IAA that makes it particularly pernicious to deal with?

Dr McCrindle. I think the method of actually repairing the interruption at the same time as dealing with the truncus is much more complex than dealing with either lesion in isolation and probably contributes to longer procedure times, which may influence subsequent outcomes. Also, the complexity of configuring the arch in the presence of truncus is much more complex, with many more sites at which things can go wrong and predispose toward subsequent reinterventions and obstructions.

Dr Mosca. It was interesting that a large VSD was protective from the need for reintervention on the LVOT. What are your thoughts concerning this observation?

Dr McCrindle. I would suspect that the presence of a small VSD and also probably the presence of VSDs that were not malaligned suggests a different disease process, and probably those patients were more likely to have associated cardiac anomalies that would have influenced the outcomes as well.

Dr Mosca. Thank you very much. I enjoyed your presentation.

Dr John E. Foker (Minneapolis, Minn). My congratulations on an excellent presentation. Although I am reluctant to advocate a retro approach, at our institution staged procedures seem to offer some advantage, particularly for the sicker infants. We have had 36 such cases without a surgical death, all done without bypass and without interruption of the cerebral circulation.

The really interesting thing, though, is that despite the theoretic objections to pulmonary arterial banding and conal-truncal malalignment, we have not had LVOT tract obstruction develop in any children after this procedure. We have had 2 who had it at birth, but none have had it develop. Of course, you do need to replace or augment the arch at about 12 years of age. Have you looked at the infants who underwent a staged repair to determine the likelihood that LVOT obstruction would develop?

Dr McCrindle. We did not particularly look at the timing as a risk factor for when the LVOT procedure was performed in relationship to the repair. I can state that 91 of the 143 patients who underwent a LVOT procedure had it performed at the time of the arch repair, but in the analysis that didn't fall out as a specific risk factor for outcomes. Given that our previous analysis focused on staging, however, this was not a focus of the current study, which emphasized interventions for LVOT obstruction and arch repair obstruction.

Dr Ross Ungerleider (Portland, Ore). You and your colleagues at the CHSS data center should be congratulated on the tireless work that you do to help us understand these complex patients. My questions are really quite simple.

First, could you tell us a little bit about the influence of DiGeorge syndrome on the outcome for patients with IAA and associated defects?

Dr McCrindle. DiGeorge syndrome was only present in about a quarter of the patients for which that datum was reported, but clearly for the era during which these patients were initially entered, the whole concept of DiGeorge and testing weren't well established. I would suspect the incidence of DiGeorge syndrome to be much higher than what we were able to report. What we are finding in our annual follow-up of survivors is that in some cases the diagnosis is being made late. Nonetheless, among those patients for whom it was documented that they had DiGeorge, it was not an incremental risk factor, particularly for mortality.

Dr Ungerleider. Second, as the periods evolved, did you notice a change in the way the LVOT problems were addressed? Were there more successes moving away from some of the older procedures to Ross-Konno type reconstructions of the LVOTs, or did you notice that there were different procedures for the arches that were becoming more successful in the later years?

Dr McCrindle. In general, we didn't really look for trends with time in the types of procedures that were performed, but we did look for trends with time in the outcomes that occurred. My impression of the data would be that the procedures to address LVOT obstruction were complex throughout the entire study period, with many novel variations for cases in which simple resections were not deemed sufficient or feasible. 


\section{Appendix A. Statistical Methods}

Flow charts were created to track patients though multiple consecutive procedures and deaths. Competing risks analyses were used to examine rates of transition from an initial state (hazard function) to the mutually exclusive time-related events of various procedures and death without that procedure. This was used to determine to proportion of patients reaching these events or states at any given time after the initial state.

Noninformative imputation based on available data was used to calculate and place the mean for missing values. Relevant missing value indicator variables were created and included in multivariable analyses to adjust for possible bias introduced by missing data.

Competing risks analyses were performed in a similar manner for each of the following: (1) from initial admission to a CHSS member institution to either death or an initial procedure aimed at addressing potential or actual LVOT obstruction, (2) from an initial procedure aimed at addressing potential or actual LVOT obstruction to either death or a subsequent similar procedure, and (3) from IAA repair to either death or a subsequent intervention for residual or recurrent obstruction at the arch repair site.
For each competing risks analysis, non-risk-adjusted nonparametric estimates for time-related freedom from death or the specified procedure were plotted with the Kaplan-Meier method. The underlying hazard function was modeled parametrically, searching for multiple phases of risk, as previously described. ${ }^{2}$ Demographic, morphologic, and procedural factors associated with each outcome were sought through multivariable analysis of these parametric models. Only variables associated with more than 5 events were included, to minimize the risk of model overdetermination. For continuous variables, various mathematic transformations were tested for optimal calibration of the relationship to risk, and the significance of various interaction terms were explored. Bootstrap bagging with cluster analysis was used to guide final variable selection and to assess reliability of variable inclusion into final multivariable models. Missing value indicator variables were entered into final multivariable models as appropriate. The multivariable models were solved in competing risks format for multiple combinations of risk factors to explore the magnitude of effect of these factors and to predict outcomes for patients with given characteristics. 
Supplementary Appendix B: TABLE 1. Patient characteristics ( $n=472$ )

\begin{tabular}{|c|c|c|c|c|}
\hline Variable & No. & Missing & Value & Deaths $(n=186)$ \\
\hline \multicolumn{5}{|l|}{ Demographic characteristics } \\
\hline Age at admission ( $d$, median and range) & 468 & 4 & 3 (birth-30) & \\
\hline \multicolumn{5}{|l|}{ Demographic characteristics } \\
\hline Age at admission ( $d$, median and range) & 468 & 4 & 3 (birth-30) & \\
\hline Birth weight $(\mathrm{kg}$, mean $\pm \mathrm{SD})$ & 190 & 282 & $3.04 \pm 0.64$ & \\
\hline Sex (female/male) & 471 & 1 & $234: 237$ & $80: 106$ \\
\hline Noncardiac anomaly (No.) & 472 & 0 & $165(35 \%)$ & $68(41 \%)$ \\
\hline DiGeorge syndrome (No.) & 472 & 0 & $86(18 \%)$ & $33(38 \%)$ \\
\hline \multicolumn{5}{|l|}{ Morphologic characteristics (No.) } \\
\hline \multicolumn{5}{|l|}{ Type of IAA } \\
\hline Type A & 468 & 0 & $130(28 \%)$ & $33(26 \%)$ \\
\hline Type B & 468 & 0 & $331(70 \%)$ & $144(44 \%)$ \\
\hline Type C & 468 & 0 & $7(1 \%)$ & $7(100 \%)$ \\
\hline \multicolumn{5}{|l|}{ Major associated cardiac anomalies } \\
\hline None-isolated VSD & 472 & 0 & $341(72 \%)$ & $121(35 \%)$ \\
\hline None-intact ventricular septum & 472 & 0 & $7(1 \%)$ & $2(29 \%)$ \\
\hline Aortopulmonary window & 472 & 0 & $20(4 \%)$ & $3(15 \%)$ \\
\hline Complete atrioventricular septal defect & 472 & 0 & $3(<1 \%)$ & $3(100 \%)$ \\
\hline Atrioventricular discordance & 472 & 0 & $2(<1 \%)$ & $1(50 \%)$ \\
\hline Double-outlet right ventricle & 472 & 0 & $8(2 \%)$ & $4(50 \%)$ \\
\hline Partial anomalous pulmonary venous return & 472 & 0 & $1(<1 \%)$ & $1(100 \%)$ \\
\hline Single ventricle & 472 & 0 & $13(3 \%)$ & $9(69 \%)$ \\
\hline Transposition of great arteries with VSD & 472 & 0 & $26(6 \%)$ & $9(35 \%)$ \\
\hline Truncus arteriosus & 472 & 0 & $51(11 \%)$ & $34(67 \%)$ \\
\hline Bicuspid aortic valve & 229 & 243 & $149(65 \%)$ & $57(38 \%)$ \\
\hline Anomalous right subclavian artery & 427 & 45 & $108(25 \%)$ & $45(42 \%)$ \\
\hline Left superior vena cava & 440 & 32 & $35(8 \%)$ & $19(54 \%)$ \\
\hline Large patent ductus arteriosus & 281 & 191 & $251(89 \%)$ & $94(37 \%)$ \\
\hline Large VSD & 392 & 80 & $322(82 \%)$ & $123(38 \%)$ \\
\hline Multiple VSDs & 383 & 89 & $31(8 \%)$ & $14(45 \%)$ \\
\hline Malalignment of VSD & 472 & 0 & $165(35 \%)$ & $74(32 \%)$ \\
\hline
\end{tabular}


Supplementary Appendix C: TABLE 2. Characteristics of IAA repair $(n=453)$

\begin{tabular}{|c|c|c|c|}
\hline Variable & No. & Missing & Value \\
\hline Age at repair ( $d$, median and range) & 452 & 1 & $7(1-60)$ \\
\hline Weight at repair $(\mathrm{kg}$, mean $\pm \mathrm{SD})$ & 365 & 88 & $3.09 \pm 0.59$ \\
\hline \multicolumn{4}{|l|}{ Concomitant procedures (No.) } \\
\hline Closure of VSD & 453 & 0 & $270(60 \%)$ \\
\hline Pulmonary artery banding & 441 & 12 & $96(22 \%)$ \\
\hline Main pulmonary artery-aorta anastomosis & 453 & 0 & $50(11 \%)$ \\
\hline Repair of truncus arteriosus & 453 & 0 & $42(9 \%)$ \\
\hline Systemic-pulmonary artery shunt placement & 453 & 0 & $34(8 \%)$ \\
\hline Mitral valve repair & 450 & 3 & $1(<1 \%)$ \\
\hline \multicolumn{4}{|l|}{ Technique of arch repair (No.) } \\
\hline \multicolumn{4}{|l|}{ Approach } \\
\hline Median sternotomy & 453 & 0 & $328(72 \%)$ \\
\hline Thoracotomy & 453 & 0 & $123(27 \%)$ \\
\hline Both sternotomy and thoracotomy & 453 & 0 & $2(<1 \%)$ \\
\hline Augmentation of aortic arch & 453 & 0 & $115(25 \%)$ \\
\hline \multicolumn{4}{|l|}{ Type of repair of IAA } \\
\hline Direct anastomosis with no patching & 453 & 0 & $267(59 \%)$ \\
\hline Direct anastomosis with patching & 453 & 0 & $125(28 \%)$ \\
\hline Interposition graft & 453 & 0 & $59(13 \%)$ \\
\hline Main pulmonary artery-aorta bypass conduit & 453 & 0 & $2(<1 \%)$ \\
\hline Use of graft material & 453 & 0 & $187(41 \%)$ \\
\hline PTFE & 58 & & \\
\hline Pulmonary artery homograft & 52 & & \\
\hline Other & 28 & & \\
\hline Pericardium & 23 & & \\
\hline Aortic homograft & 19 & & \\
\hline Xenograft & 4 & & \\
\hline Unspecified homograft & 3 & & \\
\hline \multicolumn{4}{|l|}{ Subclavian artery } \\
\hline None divided & 453 & 0 & $385(85 \%)$ \\
\hline Left divided & 453 & 0 & $39(9 \%)$ \\
\hline Right divided & 453 & 0 & $23(5 \%)$ \\
\hline Both divided & 453 & 0 & $6(1 \%)$ \\
\hline
\end{tabular}


Appendix D: Electronic Figure 1. Flow chart illustrating procedures for IAA repair, LVOT obstruction, and IAA repair reintervention and associated deaths for 472 neonates with IAA.

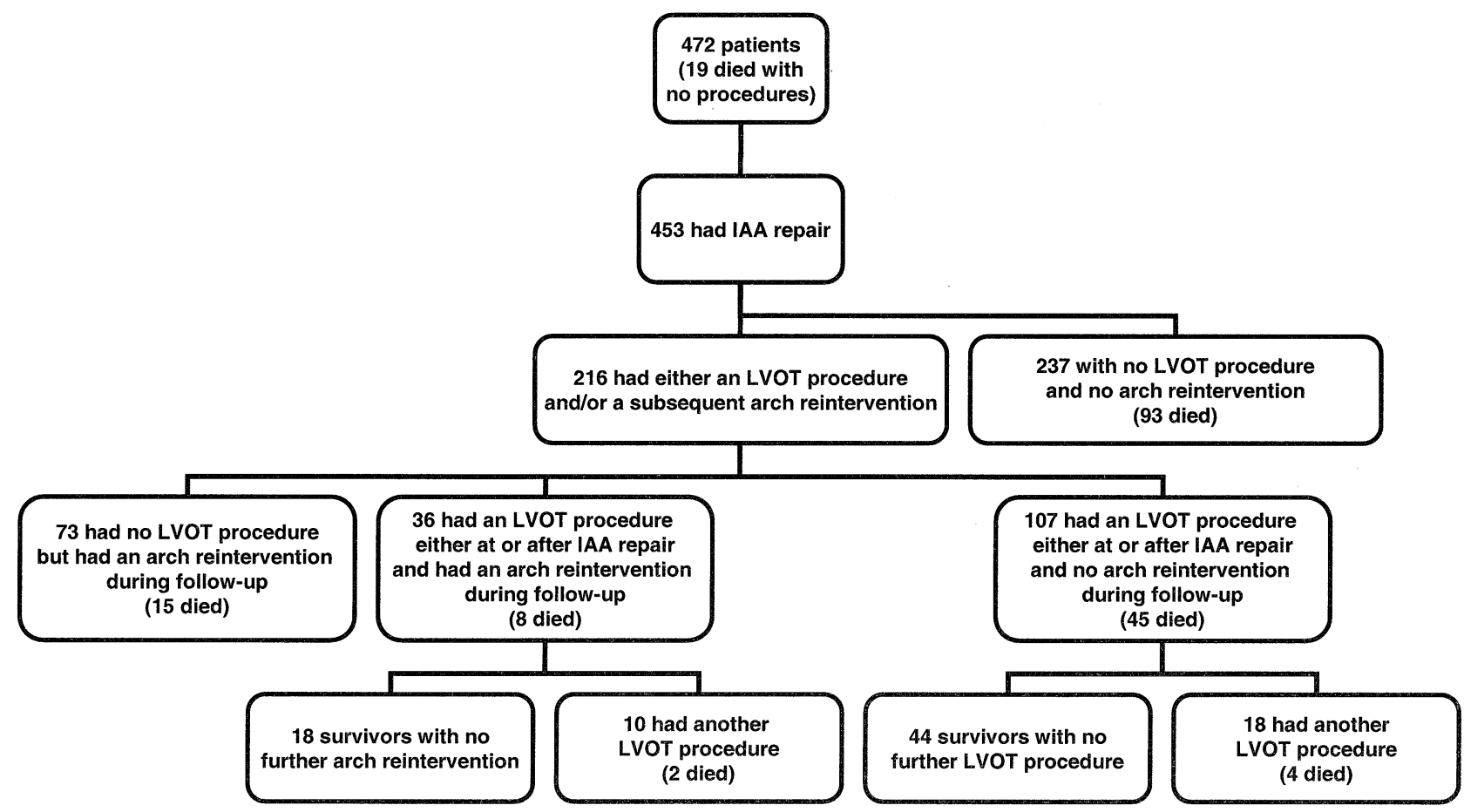




\section{Supplementary Appendix E: TABLE 3. Incremental risk factors for time-related transition from IAA repair ( $n=453$ ) to either a reintervention at the IAA repair site or death, with and without institutions as additional risk factors}

\begin{tabular}{lccc}
\hline Variable & Parameter & $P$ & Reliability \\
estimate \pm SE & value & (\%) \\
\hline
\end{tabular}

For subsequent IAA reintervention

Without institutions

Early phase

Younger and older age at admission*

Aortopulmonary window

Double-outlet right ventricle

See legend

$1.11 \pm 0.35$

.001

$65 \%$

$1.40 \pm 0.53$

.007

$75 \%$

Late phase

Repair of associated truncus arteriosus at IAA repair

Repair of IAA by method other than direct anastomosis

$2.95 \pm 0.65$

$<.001$

$60 \%$

with patch augmentation

Use of PTFE for IAA repair

$1.61 \pm 0.94$

.09

$49 \%$

$2.60 \pm 0.56$

$<.001$

$56 \%$

With institutions

Early phase

Younger and older age at admission*

Aortopulmonary window

See legend

$0.95 \pm 0.36$

$1.55 \pm 0.53$

$1.52 \pm 0.42$

$1.42 \pm 0.42$

Institution E

Institution $\mathrm{F}$

Institutions contributing $<10$ patients

$0.74 \pm 0.26$

$2.43 \pm 0.72$

$2.57 \pm 0.61$

$0.008 \pm 0.003$

.008

$<.001$

$<.001$

$0.81 \pm 0.23$

$<.001$

$0.84 \pm 0.24$

$0.56 \pm 0.22$

.01

$0.51 \pm 0.19$

.007

$0.66 \pm 0.22$

.002

$<.001$

$58 \%$

Absence of malalignment of the VSD

No augmentation of aortic arch at IAA repair

Main pulmonary artery-aorta anastomosis performed at

$0.81 \pm 0.24$

$0.007 \pm 0.003$

.03

$78 \%$

IAA repair

With institutions

Earlier date of birth†

Lower weight at IAA repair§

Truncus arteriosus

IAA type B or C

VSD size not large

$2.19 \pm 0.40$

$<.001$

$<.001$

$0.90 \pm 0.23$

.006

$0.64 \pm 0.22$

$0.65 \pm 0.32$

$$
.004
$$

$$
.04
$$

$0.60 \pm 0.22$

.008

$0.92 \pm 0.23$

$<.001$

$88 \%$

No augmentation of aortic arch at IAA repair

Main pulmonary artery-aorta anastomosis performed at

IAA repair

Institution A

$0.63 \pm 0.27$

$1.14 \pm 0.27$

.02

$0.68 \pm 0.31$

$<.001$

$68 \%$

$82 \%$

$68 \%$

$76 \%$

$57 \%$

$56 \%$

$56 \%$

Institution G (protective)

*Complex polynomial function including two terms, age at admission in days and the logarithm of age at admission. $†$ After squared transformation of the interval from study onset to patient date of birth in years. $\ddagger$ After inverse transformation of weight at IAA repair in kilograms. §After logarithmic transformation of weight at IAA repair in kilograms. 
Supplementary Appendix F: TABLE 4. Type of initial intervention aimed at potential or actual LVOT obstruction ( $n=143)$

Subsequent LVOT

Initial LVOT intervention

No. intervention

Primary cardiac transplantation

Aortic valve intervention

Transcatheter balloon valvotomy/dilatation of subaortic stenosis

Surgical valvuloplasty

Surgical valvotomy

Aortic valve replacement

Transcatheter balloon valvotomy/dilatation of subaortic stenosis

Subaortic surgical intervention

Resection of LVOT obstruction

Conal resection

Enlargement of VSD

Conal resection with VSD enlargement

Konno procedure with aortic valve replacement

Modified Konno procedure

LVOT obstruction bypass intervention

Transection of MPA with side-to-side anastomosis to ascending aorta

Transection of MPA with end-to-side anastomosis to ascending aorta

End-to-end anastomosis of MPA to descending aorta with PA banding

Creation of an MPA to ascending aorta window with PA banding

Conduit placement from MPA to descending aorta with PA banding

Left ventricle-descending aorta conduit placement

\begin{tabular}{rcc} 
No. & Died & intervention \\
\hline 2 & 1 & 0 \\
10 & & 8 \\
3 & 0 & 0 \\
1 & 1 & 0 \\
1 & 0 & 0 \\
10 & 1 & 8 \\
& 0 & \\
40 & 0 & 10 \\
20 & & 4 \\
6 & 8 & 0 \\
3 & 8 & 1 \\
5 & 3 & 2 \\
1 & 1 & 1 \\
& 0 & \\
35 & 0 & 2 \\
6 & & 0 \\
4 & 21 & 0 \\
4 & 2 & 0 \\
1 & 2 & 0 \\
1 & 3 & 0 \\
\hline
\end{tabular}

$M P A$, Main pulmonary artery; $P A$, pulmonary artery. 
Supplementary Appendix G: TABLE 5. Incremental risk factors for time-related transition from admission ( $n=472$ ) to either an initial LVOT intervention or death, with and without institutions as additional risk factors

\begin{tabular}{|c|c|c|c|}
\hline Variable & $\begin{array}{c}\text { Parameter } \\
\text { estimate } \pm \text { SE }\end{array}$ & $P$ value & $\begin{array}{c}\text { Reliability } \\
(\%)\end{array}$ \\
\hline \multicolumn{4}{|l|}{ For initial LVOT intervention } \\
\hline \multicolumn{4}{|l|}{ Without institutions } \\
\hline \multicolumn{4}{|l|}{ Early phase } \\
\hline Lower birth weight* & $3.98 \pm 1.52$ & .009 & $45 \%$ \\
\hline Single ventricle & $1.66 \pm 0.48$ & $<.001$ & $84 \%$ \\
\hline IAA type B & $0.85 \pm 0.30$ & .004 & $63 \%$ \\
\hline \multicolumn{4}{|l|}{ Late phase } \\
\hline Single ventricle & $3.12 \pm 0.84$ & $<.001$ & $33 \%$ \\
\hline Anomalous right subclavian artery & $0.74 \pm 0.35$ & .04 & $50 \%$ \\
\hline Bicuspid aortic valve & $1.32 \pm 0.70$ & .06 & $55 \%$ \\
\hline \multicolumn{4}{|l|}{ With institutions } \\
\hline \multicolumn{4}{|l|}{ Early phase } \\
\hline Lower birth weight* & $2.70 \pm 1.57$ & .09 & $55 \%$ \\
\hline Single ventricle & $1.62 \pm 0.50$ & .001 & $82 \%$ \\
\hline IAA type B & $0.82 \pm 0.30$ & .006 & $66 \%$ \\
\hline Institution A & $0.94 \pm 0.34$ & .005 & $42 \%$ \\
\hline Institution B & $0.96 \pm 0.32$ & .002 & $46 \%$ \\
\hline \multicolumn{4}{|l|}{ Late phase } \\
\hline Single ventricle & $3.14 \pm 0.79$ & $<.001$ & $33 \%$ \\
\hline Anomalous right subclavian artery & $0.77 \pm 0.34$ & .04 & $55 \%$ \\
\hline Bicuspid aortic valve & $1.31 \pm 0.68$ & .06 & $46 \%$ \\
\hline \multicolumn{4}{|l|}{ For death without initial LVOT intervention } \\
\hline \multicolumn{4}{|l|}{ Without institutions } \\
\hline \multicolumn{4}{|l|}{ Early phase only } \\
\hline Earlier date of birtht & See legend & & $98 \%$ \\
\hline Lower birth weight & See legend & & $97 \%$ \\
\hline Female sex & $0.39 \pm 0.18$ & .04 & $53 \%$ \\
\hline Younger age at admission§ & $0.26 \pm 0.11$ & .02 & $64 \%$ \\
\hline Truncus arteriosus & $1.05 \pm 0.21$ & $<.001$ & $94 \%$ \\
\hline IAA type $B$ or $C$ & $0.58 \pm 0.24$ & .02 & $81 \%$ \\
\hline \multicolumn{4}{|l|}{ With institutions } \\
\hline Earlier date of birth\| & See legend & & $97 \%$ \\
\hline Lower birth weight $\ddagger$ & See legend & & $98 \%$ \\
\hline Female sex & $0.45 \pm 0.19$ & .02 & $36 \%$ \\
\hline Younger age at admission§ & $0.26 \pm 0.11$ & .02 & $64 \%$ \\
\hline Truncus arteriosus & $1.06 \pm 0.21$ & $<.001$ & $58 \%$ \\
\hline IAA type B or C & $0.52 \pm 0.24$ & .03 & $48 \%$ \\
\hline Institution C & $0.89 \pm 0.29$ & .003 & $58 \%$ \\
\hline Institution D & $1.19 \pm 0.48$ & .02 & $50 \%$ \\
\hline
\end{tabular}

*After inverse transformation of the square of birth weight. †Complex polynomial function including two terms, interval from study onset to patient date of birth in years and the inverse transformation of this interval. \$Complex polynomial function including two terms, the logarithmic transformation and the inverse transformation of birth weight in kilograms. §After logarithmic transformation. ||Complex polynomial function including two terms, interval from study onset to patient date of birth in years after squared transformation and additionally after inverse transformation of the squared transformation. 
Supplementary Appendix H: TABLE 6. Incremental risk factors for time-related transition from an initial LVOT intervention $(n=143)$ to either a subsequent LVOT intervention or death, with and without institutions as additional risk factors

\begin{tabular}{|c|c|c|c|}
\hline Variable & $\begin{array}{c}\text { Parameter } \\
\text { estimate } \pm S E\end{array}$ & $P$ value & $\begin{array}{c}\text { Reliability } \\
(\%)\end{array}$ \\
\hline \multicolumn{4}{|l|}{ For subsequent LVOT intervention } \\
\hline \multicolumn{4}{|l|}{ Without institutions } \\
\hline \multicolumn{4}{|l|}{ Late phase only } \\
\hline Initial balloon dilatation of subaortic obstruction & $.63 \pm 0.42$ & $<0.001$ & $54 \%$ \\
\hline VSD size not large & $0.87 \pm 0.41$ & 0.04 & $63 \%$ \\
\hline \multicolumn{4}{|l|}{ With institutions } \\
\hline Initial balloon dilation of subaortic obstruction & $2.63 \pm 0.53$ & $<0.001$ & $54 \%$ \\
\hline VSD size not large & $1.20 \pm 0.44$ & 0.006 & $70 \%$ \\
\hline Institution G (protective) & $1.68 \pm 0.64$ & 0.009 & $57 \%$ \\
\hline \multicolumn{4}{|l|}{ For death without subsequent LVOT intervention* } \\
\hline \multicolumn{4}{|l|}{ Early phase only } \\
\hline Earlier date of birth $\dagger$ & $0.19 \pm 0.06$ & $<0.001$ & $71 \%$ \\
\hline Female sex & $0.61 \pm 0.29$ & 0.04 & $51 \%$ \\
\hline Absence of malalignment of VSD & $1.29 \pm 0.35$ & $<0.001$ & $76 \%$ \\
\hline Systemic-pulmonary artery shunt with initial LVOT intervention & $1.74 \pm 0.63$ & 0.006 & $49 \%$ \\
\hline Pulmonary artery band with initial LVOT intervention & $2.01 \pm 0.56$ & $<0.001$ & $51 \%$ \\
\hline Closure of VSD with initial LVOT intervention & $2.29 \pm 0.66$ & $<0.001$ & $54 \%$ \\
\hline Lower patient weight at initial LVOT intervention $\ddagger$ & $6.75 \pm 1.41$ & $<0.001$ & $81 \%$ \\
\hline $\begin{array}{l}\text { Initial LVOT procedure of main pulmonary artery-aorta anastomosis } \\
\text { with either systemic-pulmonary artery shunt or right ventricle- } \\
\text { pulmonary artery conduit (interaction term) }\end{array}$ & $0.87 \pm 0.40$ & 0.03 & $70 \%$ \\
\hline
\end{tabular}

*No institutions were significant incremental risk factors. †Defined as interval in years from January 1, 1987, to patient’s date of birth. $¥$ After inverse transformation of weight in kilograms. 